$59 N 0 \%+1327 \mathrm{G}$

Invited Papz: far SPIE Sympesum April 24-30. 1998, Santa Fe, Nim. Symposum Title: High Power Laser Ablation i998 SAND- $-98-1327 \mathrm{C}$
CONF $-980447--$

\title{
Characterization of amorphous carbon films grown by pulsed-laser deposition
}

Michael P. Siegal ${ }^{a}$, Luz J. Martinez-Miranda ${ }^{b}$, N. John DiNardo ${ }^{c}$ David R. Tallant ${ }^{a}$, J. Charles Barbour ${ }^{a}$, and Paula Newcomer Provencio ${ }^{a}$

\author{
${ }^{a}$ Sandia National Laboratories, Albuquerque, NM 87185-1421 \\ ${ }^{b}$ Dept. of Materials and Nuclear Engineering, Univ. of Maryland, College Park, MD 20742 \\ ${ }^{c}$ Dept. of Physics and Atmospheric Science, Drexel University, Philadelphia, PA 19104
}

\begin{abstract}
Amorphous carbon (a-C) films grow via energetic processes such as pulsed-laser deposition (PLD). The coldcathode electron emission properties of a-C are promising for flat-panel display and vacuum microelectronics technologies. These ultrahard films consist of a mixture of 3-fold and 4-fold coordinated carbon atoms, resulting in an amorphous material with "diamond-like" properties. We study the structures of a-C films grown at room temperature as a function of PLD energetics using x-ray reflectivity, Raman spectroscopy, high-resolution transmission electron microscopy, and Rutherford backscattering spectrometry. While an understanding of the electron emission mechanism in a-C films remains elusive, the onset of emission is typically preceded by "conditioning" where the material is stressed by an applied electric field. To simulate conditioning and assess its effect, we use the spatially-localized field and current of a scanning tunneling microscope tip. Scanning force microscopy shows that conditioning alters surface morphology and electronic structure. Spatially-resolved electron energy loss spectroscopy indicates that the predominant bonding configuration changes from predominantly 4 -fold to 3 -fold coordination.
\end{abstract}

Keywords: amorphous carbon, pulsed-laser deposition, density, electron emission

\section{INTRODUCTION}

Amorphous carbon (a-C) films with properties approaching those of diamond have been grown using energetic physical deposition methods, such as ion beam, cathodic filtered ion arc, or pulsed-laser deposition (PLD). ${ }^{1-3}$ The energetics of the film growth processes determine the ratio of 3-fold ( $\mathrm{sp}^{2}$ or graphite-like) to 4-fold ( $\mathrm{sp}^{3}$ or diamondlike) coordinated carbon atoms. The topology and ratio of these carbon bond types control the properties of the materials. Numerous papers in the literature have reported $\mathrm{a}-\mathrm{C}$ structural properties such as the bond type ratio, residual film stress, and mass density as a function of the growth energetics. ${ }^{4-12}$ Others have correlated these properties to hardness, electronic transport, dielectric permittivity, optical transparency, and cold-cathode electron emission. $^{13-18}$ Most of these studies assume that a-C film structures are essentially homogeneous in the direction perpendicular to the substrate, i.e. throughout the thickness of a given film. The greatest complexity typically assumed is the existence of a few nanometer-thick region of higher 3-fold coordinated carbon near the film surface. This assumption is consistent with a subplantation model for a-C growth. ${ }^{5,19}$ The model hypothesizes that for depositing carbon species to form 4-fold coordinated bonding, the species must grow in a state of very high local pressure. This pressure can be achieved through the creation of point defects from ion implantation into a sublayer just below the deposition surface. Since the final layer deposited does not experience further subplantation, a surface region results that is rich in 3 -fold coordinated carbon bonding.

Further author information -

M.P.S. (correspondence): Email: mpsiega@sandia.gov

DISTRISUTION OF THS DOCURENT IS UREMTED

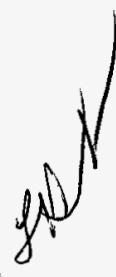




\section{DISCLAIMER}

This report was prepared as an account of work sponsored by an agency of the United States Government. Neither the United States Government nor any agency thereof, nor any of their employees, makes any warranty, express or implied, or assumes any legal liability or responsibility for the accuracy, completeness, or usefulness of any information, apparatus, product, or process disclosed, or represents that its use would not infringe privately owned rights. Reference herein to any specific commercial product, process, or service by trade name, trademark, manufacturer, or otherwise does not necessarily constitute or imply its endorsement, recommendation, or favoring by the United States Government or any agency thereof. The views and opinions of authors expressed herein do not necessarily state or reflect those of the United States Government or any agency thereof. 
There has been much theoretical and computational study performed to predict the topographical nature of the 3 -fold and 4-fold coordinated carbon atoms that are representative of a-C structures. ${ }^{20-23}$ Two independent, recently published, fully self-consistent first-principles structure calculations using a fixed a-C mass density $\sim 3.0 \mathrm{~g} / \mathrm{cm}^{3}$ find that the materials likely consist of a mixture of the two bond types connected by single, double, and conjugated bonds in a strained network of rings both smaller and larger than the six-fold rings that characterize crystalline carbon. $^{20,21}$ Radial distribution functions from these resulting structures superbly match experimental results. ${ }^{21}$ This agreement suggests that the amorphous structures determined by these calculations are likely to be fairly representative of real a-C films. This is important since no physical experiment has been identified that is capable of direct observation of carbon-carbon bonding in an amorphous material.

Growth of a-C films occurs at room temperature in high-vacuum, lending itself to nearly any substrate material. Unfortunately, it also grows with large residual compressive stresses, on the order of several GPa, that appear to be a direct result of the energetic growth processes. ${ }^{19}$ These very high stress values limit the thickness $(100-200 \mathrm{~nm})$ to which a-C films can be grown due to adhesion failure at the film-substrate interface. It has recently been shown that these stresses can be completely relieved to negligible levels by simple thermal anneals in inert ambients at temperatures well below the decomposition of a-C into more graphitic-like material. ${ }^{24}$ This postannealing process does change the structural properties of a-C films in a subtle manner. In this paper, we will show the variations that occur in density, bond configurations and optical transparency as a result of growth energetics. The changes occurring as a result of thermal post-processing are presented elsewhere. ${ }^{25}$

Perhaps the most intriguing property demonstrated for a-C films is cold-cathode electron emission under relatively low electric-field conditions. This property is critical to the development of entire new technologies based on field emission devices such as flat-panel displays with picture quality comparable to cathode ray tubes, and vacuum microelectronics. ${ }^{26}$ The latter would include the development of devices such as vacuum tubes compatible in size and chemistry with MOSFET technologies. Vacuum tube electronics have the advantage of working at high power levels due to the lack of a dielectric material that can breakdown under high field conditions.

While an understanding of the electron emission mechanism in a-C films remains elusive, the onset of emission is typically preceded by "conditioning" where the material is stressed by an applied electric field. ${ }^{16,27}$ To simulate conditioning and assess its effect, we use the spatially-localized field and current of a scanning tunneling microscope tip. ${ }^{28}$ Scanning force microscopy shows that conditioning alters surface morphology and electronic structure. Spatially-resolved electron energy loss spectroscopy indicates that the predominant bonding configuration changes from predominantly 4-fold to 3-fold coordination.

\section{EXPERIMENT}

We use $248 \mathrm{~nm}$ radiation from a $\mathrm{KrF}$ excimer laser to ablate carbon species from a pyrolytic graphite target in a vacuum chamber with base pressure $<10^{-7}$ Torr. The carbon specie is deposited onto a rotating $\mathrm{Si}(100)$ substrate maintained at ambient temperature (below $50^{\circ} \mathrm{C}$ ). The target to substrate distance is $\sim 5$ inches. The rotating target is positioned slightly off-axis from the central portion of the plume, allowing uniform a-C film growth on substrates up to 4 inches in diameter. Laser pulses (duration $\sim 17 \mathrm{~ns}$ ) are focused on to a rectangular spot of the center of the rotating target to prevent excessive cratering with an area $\sim 0.01 \mathrm{~cm}^{2}$. Repetition rates from $10-20 \mathrm{~Hz}$ are typically used. Energy density is varied from $5-125 \mathrm{~J} / \mathrm{cm}^{2}$, achieving a-C deposition rates $5-10 \mathrm{~nm} /$ minute. Films ranging from $50-110 \mathrm{~nm}$ in thickness are grown for study.

Atomic force microscopy (AFM) is used to determine film morphology. Cross-sectional high-resolution transmission electron microscopy (HRTEM) is performed to observe a-C density-related contrast variations in the films in the direction perpendicular to the substrate. The JEOL 2010, which has a very low spherical aberration, is used in conjunction with a Gatan 694 slow-scan camera coupled with digital processing capabilities. Images are processed and analyzed using Gatan's Digital Micrograph software. Rutherford backscattering spectrometry (RBS), using $3.5 \mathrm{MeV} \mathrm{He}$ ions yields precise carbon atom areal density. Together with HRTEM thickness measurements, very accurate mass density measurements are made. Density is also determined by fitting $\mathrm{x}$-ray reflectivity (XRR) data to the Fresnel scattering equations. This nondestructive measurement also yields information on the film thickness, surface roughness, and the presence of quasi-periodic features on the order of tens of nanometers in extent within the films. XRR is performed at the National Synchrotron Light Source (Brookhaven National Laboratory), beamline X22A, using $1.20373 \AA(10.3 \mathrm{keV})$ x-rays. The experimental full width half maximum resolution, 
achieved with a $\mathrm{Si}(111)$ monochromator and $\mathrm{Si}(111)$ analyzer, is $\Delta \mathrm{E} / \mathrm{E} \sim 1 \times 10^{-4}$. Raman spectroscopy is used to study the nature of the 3-fold coordinated carbon atoms within the a-C film structures. Raman spectra are obtained using a triple spectrograph and a liquid-nitrogen cooled charge-coupled detector with $6 \mathrm{~cm}^{-1}$ resolution. ${ }^{29}$ Raman scatter is excited using the $514.5 \mathrm{~nm}$ wavelength (green light) of an argon ion laser.

Finally, we studied the initiation of electron emission in a-C films using scanning nanoprobe techniques. Emission was simulated using the high, spatially localized electric field and electron flux from a scanning tunneling microscopy (STM) tip. This results in the creation of nanostructures on the order of the tip dimension $(\sim 100 \mathrm{~nm}$ in extent). We study the changes in morphology and electronic structure using scanning force microscopy (SFM) and spatially-resolved electron-energy-loss spectroscopy (EELS). We infer that these changes are analogous to those that occur during the conditioning process for electron emission. ${ }^{28}$

\section{RESULTS}

Amorphous carbon films grown by PLD are very smooth and mirror-like in appearance. Under optical or scanning electron microscopes, no features are observable. The atomic-scale resolution of nanoscopy such as in AFM or STM is required to observe the morphology of a-C films. Fig. 1 shows an AFM image of an a-C film grown at $45 \mathrm{~J} / \mathrm{cm} 2$. The root-mean-square roughness of the film surface is less than $0.6 \mathrm{~nm}$. The smooth morphology and highly-reflective quality exists for all the films grown for this experiment over the range of $5-125$ $\mathrm{J} / \mathrm{cm}^{2}$. The image on the left is an AFM image with the gray scale corresponding to variation in the surface height over a range of $0.6 \mathrm{~nm}$. The image on the right is a tilted view of the same image to enhance the ability to easily observe the variations in surface morphology.
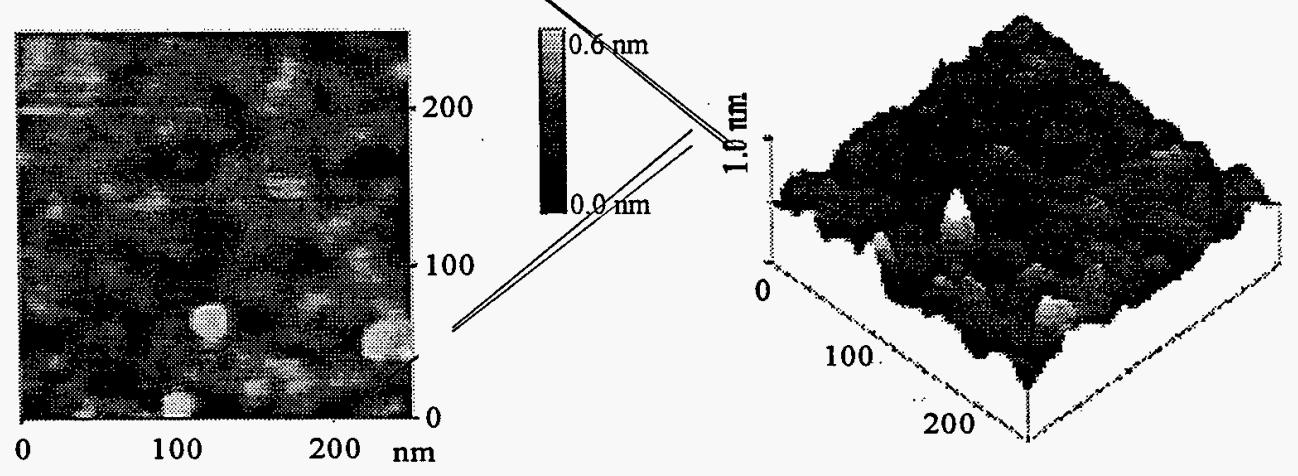

Figure 1: AFM image of a-C film morphology. On the left is a standard plan-view image. This image is tilted to provide a better view of the surface roughness on the right.

\subsection{HRTEM and RBS}

Figure 2 is a cross-sectional HRTEM image of a nominally $50 \mathrm{~nm}$ thick a-C film grown at $11 \mathrm{~J} / \mathrm{cm}^{2}$. A thin gold coating was sputter deposited onto the film surface to easily denote its location. From this image we can precisely measure the total film thickness to be $43 \mathrm{~nm}$. RBS analysis yielded a carbon areal density of $6.59 \times 10^{17}$ ( \pm $5 \%) \mathrm{C}$ atoms $/ \mathrm{cm}^{3}$. This gives an average film mass density of $3.06 \mathrm{~g} / \mathrm{cm}^{3}$. However, this simple analysis assumes that the a-C film is homogeneous in density as a function of depth below the surface. Fig. 2 shows that this is not the case. Clearly, thin layers of varying contrast exist within the a-C film at both its surface and substrate interface. The brighter region at the film surface probably results from the lower density a-C material expected at the end of the film growth process, consistent with the subplantation model. ${ }^{5,19}$ This layer of film has not experienced the same localized high pressure as the bulk of the material. The bright layer at the film-substrate interface is also likely to represent a lower density material than the bulk. TRIM code calculations find that for carbon ion implantation energies up to $400 \mathrm{eV}$, the intermixing of carbon and silicon atoms is $\leq 0.5 \mathrm{~nm}^{30}$ Therefore, a change in chemical composition at the $\mathrm{Si}-\mathrm{C}$ interface is less relevant to the formation of the observed interfacial region which is $\sim 2 \mathrm{~nm}$ 
in width. The nature of this interfacial region becomes more complex as the energetics of carbon deposition are increased. We report these results in greater detail elsewhere. ${ }^{31}$

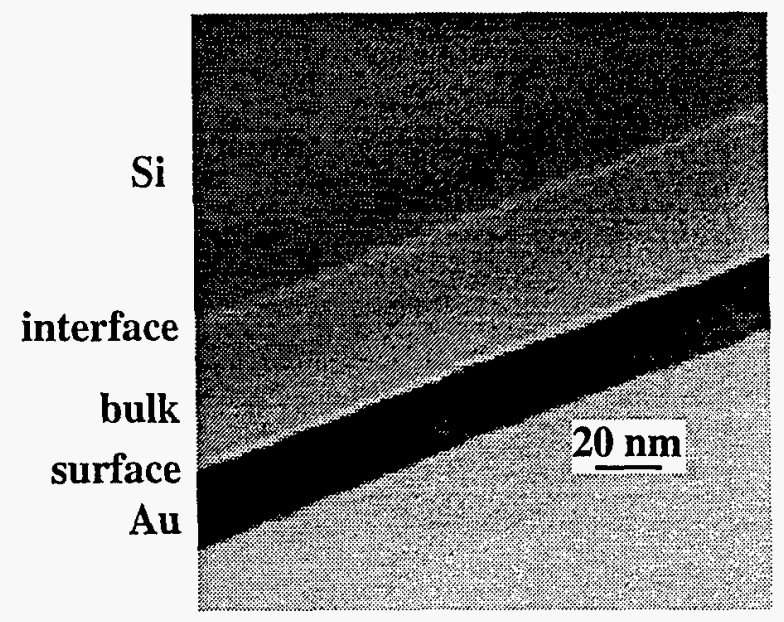

Figure 2:

HRTEM image of a $43 \mathrm{~nm}$ thick a-C film grown on $\mathrm{Si}(100)$ at $11 \mathrm{~J} / \mathrm{cm}^{3}$.

Our HRTEM study of films grown at 11,27 , and $45 \mathrm{~J} / \mathrm{cm}^{2}$ shows that the $\mathrm{Si}(100)$ substrate - carbon film interface is relatively sharp. There is no evidence for any crystallization within the films or any interfacial siliconcarbide formation. By assuming that the brightness intensity of each layer within a given HRTEM image is linearly dependent upon the density, then we can estimate the density of each layer of each film. While this approximation may be relatively crude, given the relative thickness of each layer, it is unlikely to result in a major error in the calculated density of the main bulk layer of a film. We performed these measurements using standard image analysis software and find that the densities of the three films studied range from 3.13 to $3.35 \mathrm{~g} / \mathrm{cm}^{3}$, increasing with increasing growth energetics. ${ }^{31}$ These densities are significantly higher than those reported in most other studies for films believed to have very high 4-fold coordinated $C$ atom contents $\geq 80 \%{ }^{4,6,7,32-35}$ However, it should be noted that previous reports of a-C film density were not directly measured, but rather inferred by fitting data from another measurement, such as transmission K-edge EELS, to a model dependent upon density for full simulation. The results from this measurement, which include a precise measurement of a-C thickness by HRTEM and a precise measure of atomic areal density by RBS, are likely to be the most accurate performed to date.

It is also worth noting that the majority of the computational work published to date has concentrated on a-C densities near $3.0 \mathrm{~g} / \mathrm{cm}^{3}$. The first principals studied discussed earlier found that the 4-fold coordinated carbon content is closer to $70 \%$, well below the previous experimental estimations. ${ }^{20,21}$ Assuming that K-edge EELS experiments are somewhat accurate in determining the ratio of bond types, then it is likely that the mass densities of the materials grown elsewhere and studied are closer in actual density to the values we report here. Hence, the computational studies are actually consistent with the reality of a lower density carbon material, i.e. based on the work presented here, it appears that the theoretical studies were performed on materials relatively far from "diamond-like", despite their seemingly high density. However, such low-density a-C films may be of great technological importance; films grown at the lowest PLD energy conditions have been shown to have lower turn-on fields for cold-cathode electron emission. ${ }^{16}$

\subsection{X-ray reflectivity}

$\mathrm{XRR}$ is a non-destructive method used to determine film thickness, density, roughness, and the presence of quasi-periodic features within thin films. XRR spectra are fit to the Fresnel interference equations which describe optical reflectivity. ${ }^{11,36,37}$ In general, thickness is related to the oscillation frequency in the reflectivity signal. Surface roughness is related to the exponential decay of the signal. Small angle Bragg diffraction peaks yield information on the presence of quasi-periodic structures within the films. Film density is related to the critical angle of total reflectivity.

The XRR spectra from the same three a-C films studied above $500 \AA$ grown with laser energy densities of 11 , 27 and $45 \mathrm{~J} / \mathrm{cm}^{2}$ are shown in fig. 3. A simple analysis of the oscillation widths from these XRR spectra, assuming a single layer a-C film, yields thickness measurements of 39,49 and $57 \mathrm{~nm}$, respectively, in good agreement with the 


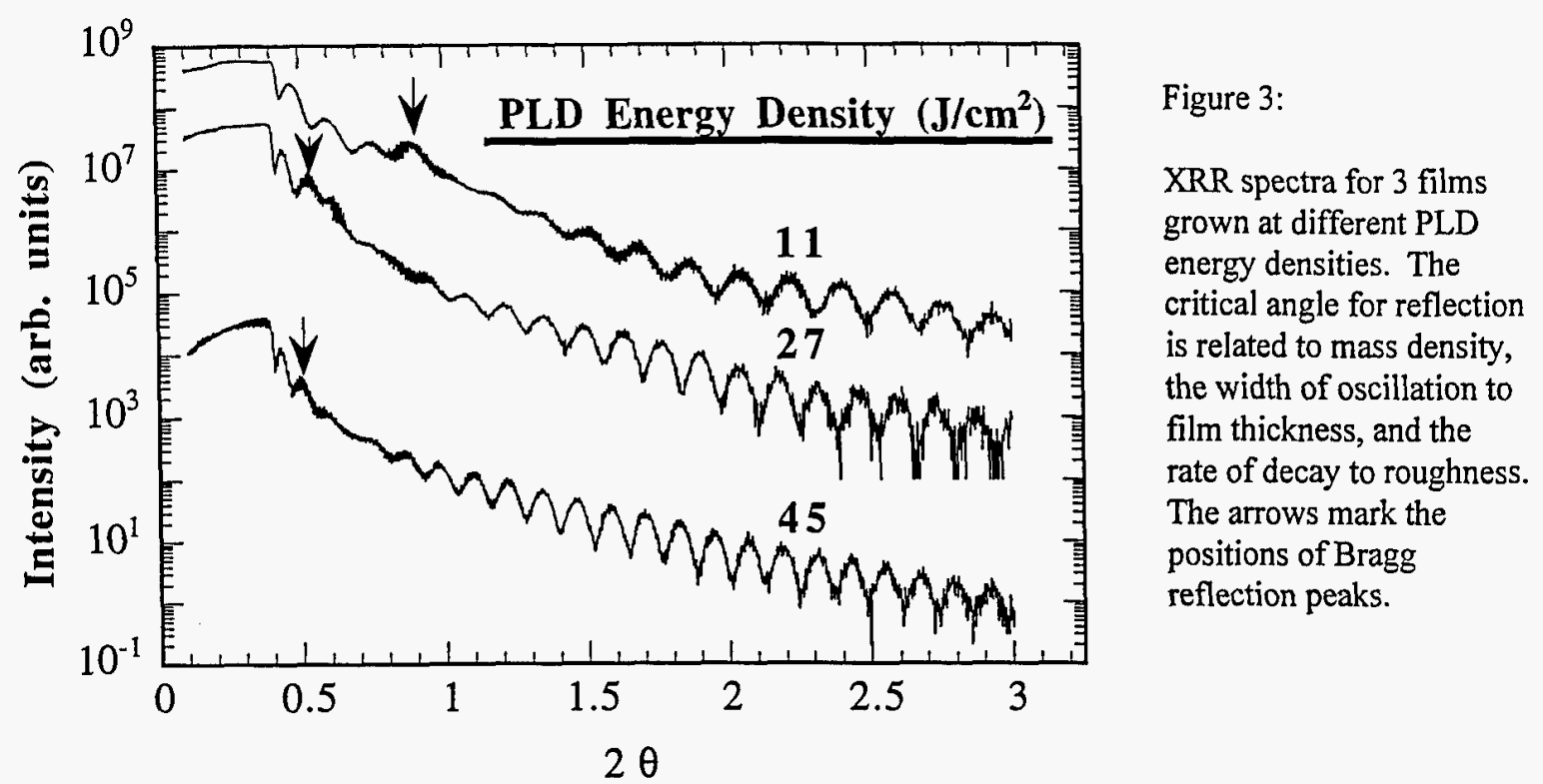

HRTEM measurements. In addition to the XRR periodic oscillations which are proportional to the film thickness, these spectra show Bragg scattering peaks, marked with arrows, near $2 \theta=0.9^{\circ}$ for the $11 \mathrm{~J} / \mathrm{cm}^{2}$ film, and near $2 \theta=$ $0.5^{\circ}$ for the 27 and $45 \mathrm{~J} / \mathrm{cm}^{2}$. These small angle peaks relate to a quasi-periodic array of scattering sites spaced 8 to $15 \mathrm{~nm}$ apart increasing as a function of growth energy. These scattering sites must represent structure(s) located at the film-substrate interface, within the film or at the film surface. The layer thicknesses determined from highresolution TEM do not correlate with these values. ${ }^{12,30}$ Additional structural characterization is being performed to help identify the source of these features.

Film density is primarily related to the critical angle of reflection. A more complete analysis, where we fit the Fresnel equations to 3 or 4 layer structural models, depending on the TEM information, will be presented elsewhere. ${ }^{12}$ For this study we fit the Fresnel equations to a simple single a-C layer film. The resulting densities calculated are remarkably similar to the precise HRTEM/RBS measurements presented above, with values ranging from 3.0 to $3.38 \mathrm{~g} / \mathrm{cm}^{3}$. This is again consistent with an increasing ratio of 4-fold coordinated carbon atoms with an increasing energetics of a-C deposition. The densities of crystalline diamond and graphite are 3.51 and $2.25 \mathrm{~g} / \mathrm{cm}^{3}$, respectively. The density of glassy carbon, or nanocrystalline graphite can be as low as $1.8 \mathrm{~g} / \mathrm{cm}^{3}$. It is expected that pure amorphous diamond will be less dense than crystalline diamond (easily understood since amorphous or highly disordered materials cannot be packed as tightly as an ordered crystal). Therefore, it is not reliable to calculate the precise 3-fold to 4 -fold coordinated $\mathrm{C}$ atom ratio based on a linear interpolation of density using the values for both graphite and diamond. However, since the density associated with 4-fold coordination is higher than that associated with 3-fold coordination, any increase in film density is correlated with an increase in 4-fold coordinated $\mathrm{C}$ atom content.

The surface roughness of these films ranges from 0 to $1 \mathrm{~nm}$. However, it cannot be stated that roughness is increasing strictly with PLD growth energy since the films monotonically increase in thickness with energetics as well. Nevertheless, it is meaningful to point out that such minor surface roughness is consistent with the subnanometer rms roughness measured in these films by AFM as shown in figure 1.

\subsection{Raman spectroscopy}

Figure 4 shows normalized Raman spectra for $\sim 100 \mathrm{~nm}$ thick a-C films grown with laser energy densities of $5,10,15,20,25,50$ and $125 \mathrm{~J} / \mathrm{cm}^{2}$. The flat-topped feature occurring between 925 and $1025 \mathrm{~cm}^{-1}$ is a second-order phonon band from the silicon substrate. Note that the intensity of this band increases, implying that the films become more transparent, or diamond-like, with increasing PLD growth energy. The broad band between 1000 and $1800 \mathrm{~cm}^{-1}$ and centered nominally at $1550 \mathrm{~cm}^{-1}$ is due to resonantly-enhanced vibrational modes of 3-fold coordinated carbon. ${ }^{14,38,39}$ Note that the carbon bands tend to narrow and shift to higher frequency with increasing deposition energy. Prior to normalization (not shown), the integrated intensity of this broad band decreases as the 
PLD growth energy increases. These trends are associated with a decrease in $\pi$-bonded structures in the film. Raman scatter from 4-fold coordinated carbon atom bonds, those exhibiting $\sigma$-like hybridization, occurs below the crystalline diamond vibrational frequency of $1330 \mathrm{~cm}^{-1}$. However, due to the lack of resonant-enhancement, Raman scatter from $\sigma$-bonding cannot be observed in the presence of $\pi$-bonds. Raman scattering using ultraviolet radiation reduces the resonant-enhancement from $\pi$-bonds and was recently shown to be an effective tool for studying $\sigma$ bonds. ${ }^{14,38,39}$

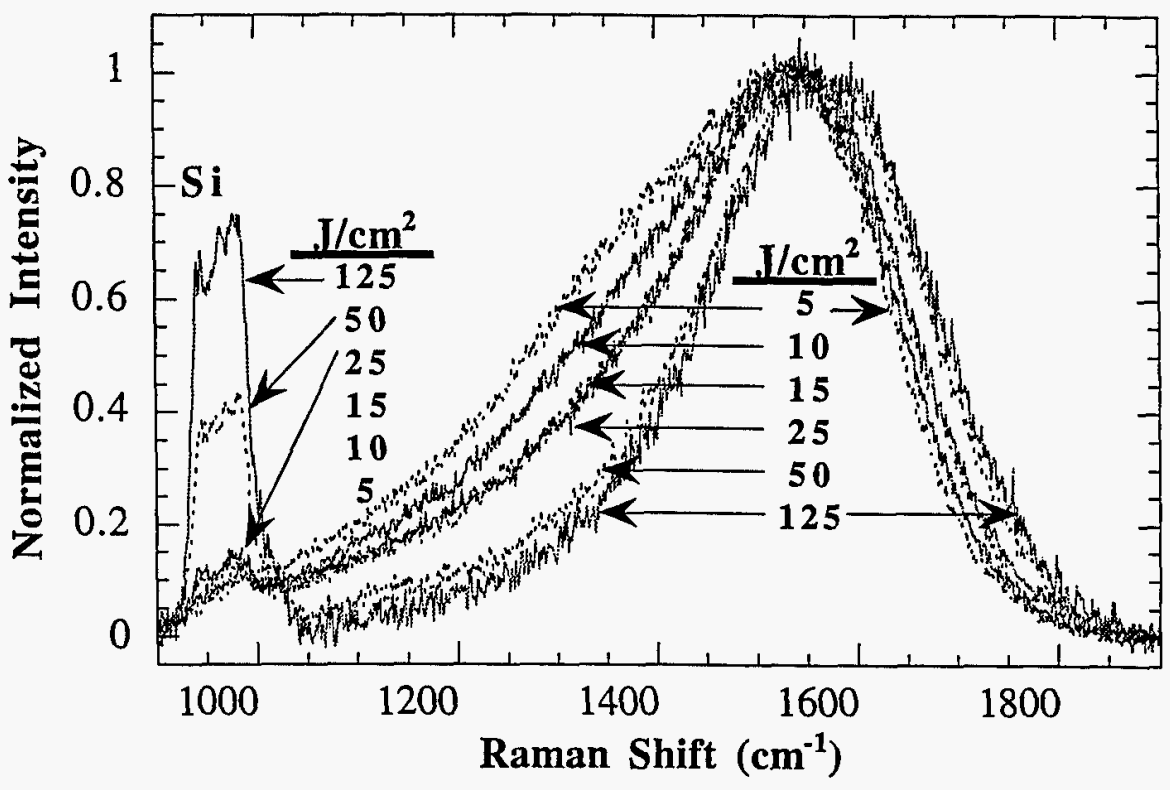

Figure 4: Raman spectra collected for $100 \mathrm{~nm}$ thick a-C films grown at PLD energies ranging from $5-125 \mathrm{~J} / \mathrm{cm}^{2}$.

The rich (and nearly continuous) variety of 3-fold and 4-fold coordinated structures predicted by theoretical calculations suggest that assignment of vibrational frequencies to specific structures may be very difficult. In the most general terms, the intensity of the Raman signal from a-C and the breadth of vibrational frequencies measured both decrease as a function of increasing growth energetics. This correlates with the fact that 3-fold coordinated carbon atoms, the sole source of the Raman signal, become less prevalent as film density increases. Various models can be used to attempt to identify carbon bonding structures that would vibrate in the manner measured in the data presented in fig. 4. In an earlier paper, we described a simple procedure for the fitting of a-C spectra into two contributions based on the active vibrational modes of disordered nanocrystalline graphite, or glassy carbon, at 1350 and $1581 \mathrm{~cm}^{-1}$. ${ }^{40}$ More recently, Doyle and Dennison calculated vibrational frequencies for idealized 5-, 6- and 7membered $\pi$-bonded carbon rings. ${ }^{41}$ While the theoretical work does not suggest that such idealized ring structures are likely to occur in metastable a-C materials ${ }^{20,21}$, it is believed that vibrational shifts due to ring distortions act mainly to broaden the range of frequencies rather than to significantly shift the average vibrational frequency for a given ring structure. ${ }^{12}$ Hence, we can attempt to interpret the Raman spectra along this somewhat more specific description. The frequencies relevant to our range of collected data are $1569 \mathrm{~cm}^{-1}$ (6 C-atom ring), $1529 \mathrm{~cm}^{-1}$ (5 and $7 \mathrm{C}$-atom rings), $1444 \mathrm{~cm}^{-1}$ (5 C-atom ring), $1360 \mathrm{~cm}^{-1}$ (6 C-atom ring), $1303 \mathrm{~cm}^{-1}$ (7 C-atom ring) and $1100 \mathrm{~cm}^{-1}$ (5C-atom ring). We find that the $1360 \mathrm{~cm}^{-1}$ frequency is never needed to fully deconvolute a spectrum. This band is related to the stacking of 6-membered rings in graphite-like structures, which probably do not exist in high density a-C, whereas the $1569 \mathrm{~cm}^{-1}$ band is dominant when 6-membered rings are relatively isolated or present in single, small sheets. Further, we note that the $1529 \mathrm{~cm}^{-1}$ frequency is representative of a-C material grown near the Si substrate/film interface, and therefore, observable only for films considerably thinner than $100 \mathrm{~nm}^{12}$. The remaining four frequencies are used to fit the data presented in fig. 4 . The excellent quality of these fits are shown elsewhere. ${ }^{12}$.

Figure 5 shows the fractional contribution of each vibrational frequency to the entire carbon portion of the Raman spectra as a function of PLD growth energy. For films grown at the lowest energy densities, the dominating frequency is at $1444 \mathrm{~cm}^{-1}$, possibly resulting from five-membered ring structures. This contribution decreases 


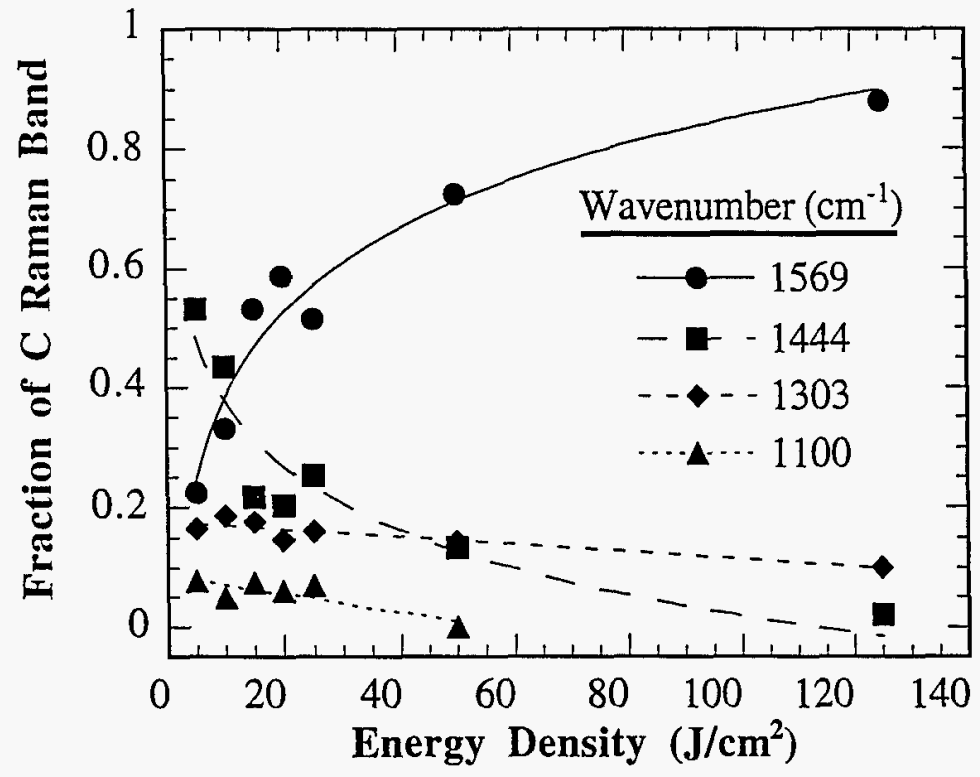

Figure 5:

The contribution of the carbon band Raman signal from Gaussians centered at frequencies based upon five-, six-, and seven-membered 3 fold coordinated carbon atom ring structures.

monotonically as a function of increasing growth energy. Contribution from the $1569 \mathrm{~cm}^{-1}$ frequency, perhaps resulting from six-membered rings, dominates with increasing growth energy. Since the overall proportion of 3-fold coordinated carbon atoms also decreases with increasing growth energy, it appears that flat sheets of six-membered rings are favored when the overall proportion is low, and the 3-fold structures are smaller and more isolated. At low deposition energies, with a higher proportion of 3-fold coordinated carbon, structures are likely to be larger and more densely packed, resulting in the incorporation of five- and seven-membered rings, similar to fullerene structures, imparting curvature to their surfaces. This behavior can be rationalized by considering the alternative to an increase in the proportion of five- and seven-membered rings with increasing 3-fold coordinated carbon. Sheets of (flat) six-membered rings, present in high density would be expected to stack, forming graphite-like structures with a distinctive Raman band signal not observed in these a-C films. Contributions from the 1303 and $1100 \mathrm{~cm}^{-1}$ frequencies are minor, and appear to decrease slightly with increasing growth energy. In general, analysis of the Raman spectra suggest the possibility that the 3-fold coordinated carbon portion of a-C films tends to become dominated by six-membered carbon rings with increasing growth energy. ${ }^{12,} 38$ The increasing presence of these structures in the 3 -fold coordinated portion of a-C correlates with the increased optical transparency of the films (seen via the Si-band in fig. 4) at higher PLD growth energies.

\subsection{Electron emission from atom probe spectroscopies}

The initiation of electron emission from high-density a-C films typically requires an electrical "conditioning" process where a voltage is applied between the film and an anode to set up an electric field. Ramping the voltage at high rates produces microdischarges that cause significant morphological crater damage. ${ }^{16,27}$ Low ramp rates are used to initiate emission without any observable morphological damage using scanning electron microscopy (SEM); however, reduced secondary electron emission occurs in the emitting regions. ${ }^{16,18}$ The threshold, or turn-on, field for emission current in the initial voltage ramp is typically $>50 \%$ higher than that required after subsequent voltage ramps. We studied the morphological and electronic structure changes using nanoprobe techniques to elucidate the nature of this conditioning process. ${ }^{28}$

High-density a-C films, which clearly must consist of very high fractions of 4-fold coordinated carbon atoms, are also good electrical insulators with dielectric permittivity constants $\sim 5-7 \varepsilon_{0} .{ }^{17}$ This leads to electric charging issues for using scanning tunneling microscopy. So for this study, a-C films $\sim 25 \mathrm{~nm}$ thick were used. The conditioning process was simulated with an STM operating in air, by ramping the STM tip bias from -10 to $+10 \mathrm{~V}$, with the feedback control disabled, at regular points of a $10 \times 10 \mu \mathrm{m}^{2}$ grid over a fresh surface region. An STM image obtained after the conditioning scan is shown in fig. 6 . The nearly complete grid pattern is the result of the conditioning process. Representative $\mathrm{I}-\mathrm{V}$ characteristics indicate that when the majority of nanostructures are 


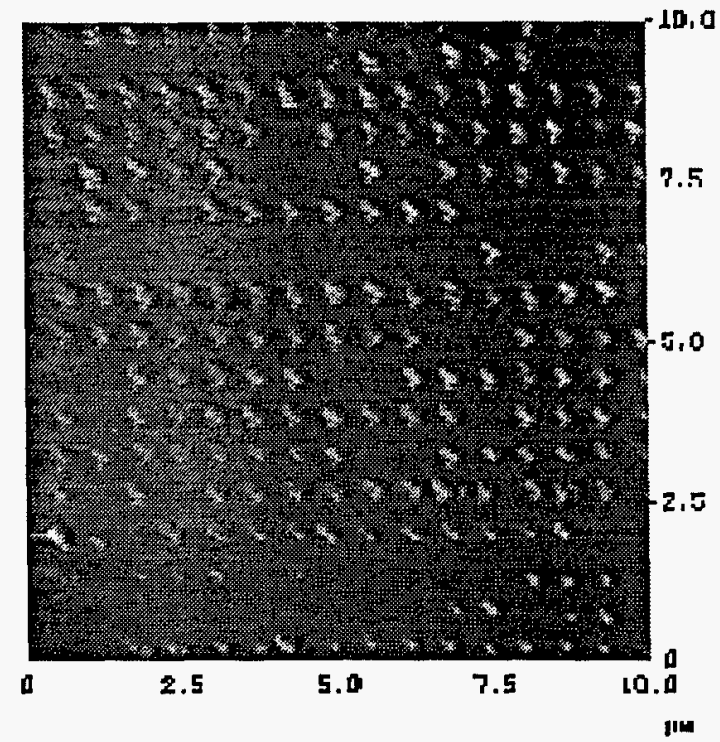

Figure 6:

STM image of a-C film after electrical conditioning over a 10 $\mathrm{x} 10 \mu \mathrm{m}^{2}$ grid.

formed, the tip-to-sample current reaches a value $\geq 100 \mathrm{nA}$. When no change occurs, a significantly reduced forward current is observed. It appears that a sufficiently high electron flux is a key factor to create a change, likely the result of a breakdown in the resistance at the sited where the elevated field is applied. ${ }^{28}$

Surface chemical composition and near-surface hybridization were probed using spatially-resolved Auger electron and EELS spectroscopies. The conditioned regions could be identified by the reduced secondary emission in the SEM mode. Auger found no evidence of impurities $(<0.02 \mathrm{~mL}$ sensitivity) in these regions. Therefore, the transfer of metal atoms from the tip can be discounted as a possible origin of the observed modification. Furthermore, conditioning the surface by STM in ultrahigh vacuum results in similar modifications, excluding the possibility of STM-induced chemical reaction of surface contaminants. ${ }^{28}$

Transmission EELS has been used in a number of studies to distinguish 3-fold and 4-fold coordinate carbon regions with subnanometer resolution. ${ }^{4}$ We used spatially resolved reflection EELS on a modified region to measure the hybridization of the near-surface carbon by analyzing shifts in the energy of the surface plasmon loss. This analysis is presented in detail elsewhere. ${ }^{28}$ Briefly, we are able to correlate plasmon shift with the STM modified regions, like those shown in fig. 6 . This shift corresponds to an increase of 3 -fold coordinated carbon species in a-C films resulting from electron emission induced surface modifications. This change in bond configurations is also consistent with the raised nanostructures evident in fig. 6; lower density 3-fold coordinated carbon material needs to increase its volume.

Demonstration that a-C films with high 4-fold coordination transform into 3-fold coordinated materials is consistent with the growing experimental evidence in the literature that diamond-like carbon materials are not ideal for cold-cathode emission applications. Earlier work in our own laboratory showed that the turn-on fields for emission decrease with decreasing PLD growth energy. ${ }^{16}$ Recent work in carbon nanotubes has found that these purely $\mathrm{sp}^{2}$-bonded carbon atoms are superb emitters. ${ }^{42-44}$ Finally, Coll and coworkers have identified a novel topological form of $\mathrm{sp}^{2}$-bonded carbon films that are "nano-coralline" in appearance from SEM imaging. ${ }^{45}$

\section{SUMMARY}

This work shows that a-C films grown by highly-energetic pulsed-laser deposition have mass densities ranging from 85 to $96 \%$ that of crystalline diamond. These densities have been accurately determined by direct measurement of film thickness and carbon atom areal density. We have confirmed these results by inference with $x-$ ray reflectivity measurements that have been carefully fitted to the Fresnel scattering equations. This validation of our XRR results provides confidence to use this non-destructive measurement technique to routinely measure the mass density of amorphous carbon, and to also extract other important properties such as film thickness and surface roughness. Atomic force microscopy finds that these very "diamond-like" a-C films are ultra-smooth on the order 
of $<1 \mathrm{~nm}$. Nano-probe microscopies and spectroscopies have helped elucidate upon the nature of electron emission in these materials, demonstrating for the first time that the initiation of electron emission results in a chemical and structural modification in the films to a less "diamond-like" state.

\section{ACKNOWLEDGEMENTS}

The authors thank J. P. Sullivan, E. B. Stechel, and P. A. Schultz for helpful discussions, T. W. Mercer for performing the nanoprobe measurements, and T. A. Friedmann for providing the amorphous-carbon films. Sandia is a multiprogram laboratory operated by Sandia Corporation, a Lockheed Martin Company, for the U. S. Department of Energy under Contract DE-AC04-94AL85000.

\section{REFERENCES}

1. S. Kasi, H. Kang, and J. W. Rabalais, "Chemically bonded diamondlike carbon from ion-beam deposition", Phys. Rev. Lett. 59, pp. 75-78, 1987.

2. P. J. Martin, S. W. Filipxzuk, R. P. Netterfield, J. S. Field, D. F. Whitnall, and D. R. McKenzie, "Structure and hardness of diamond-like carbon films prepared by arc evaporation", J. Mat. Sci. Lett. 7, pp. 410-412, 1988.

3. S. S. Wagal, E. M. Juengerman, and C. B. Collins, "Diamond-like carbon films prepared by pulsed-laser deposition", Appl. Phys. Lett. 53, pp. 187-188, 1988.

4. J. Kulik, Y. Lifshitz, G. D. Lempert, J. W. Rabalais, and D. Marton, "Electron-energy-loss spectroscopy of mass-selected ion-beam-deposited diamondlike carbon", J. Appl. Phys. 76, pp. 5063-5069, 1994.

5. D. R. McKenzie, D. Muller, and B. A. Pailthorpe, "Compressive-stress-induced formation of thin-film tetrahedral amorphous carbon", Phys. Rev. Lett. 67, pp. 773-776, 1991.

6. E. Grossman, G. D. Lempert, J. Kulik, D. Marton, J. W. Rabalais, and Y. Lifshitz, "Role of ion energy in determination of the sp3 fraction of ion beam deposited carbon", Appl. Phys. Lett. 68, pp. 1214-1216, 1996.

7. Z. Y. Chen, Y. H. Yu, J. P. Zhao, X. Wang, X. H. Liu, and T. S. Shi, "Determination of the $\mathrm{sp}^{3} / \mathrm{sp}^{2}$ ratio in amorphous carbon films by effective medium approximation", J. Appl. Phys. 83, pp. 1281-1285, 1998.

8. M. Chhowalla, C. A. Davis, M. Weiler, B. Kleinsorge, and G. A. J. Amaratunga, "Stationary carbon cathodic arc: plasma and film characterization", J. Appl. Phys. 79, pp. 2237-2244, 1996.

9. H. C. Ong and R. P. H. Chang, "Effect of laser intensity on the properties of carbon plasmas and deposited films", Phys. Rev. B, 55, pp. 13213-13220, 1997.

10. M. P. Siegal , T. A. Friedmann, S. R. Kurtz, D. R. Tallant, R. L. Simpson, F. Dominguez and K. F. McCarty, "Structural and electrical characterization of highly-tetrahedral-coordinated diamond-like carbon films grown by pulsed-laser deposition", Mat. Res. Soc. Symp. Proc. 349, pp. 507-512, 1994.

11. L. J. Martinez-Miranda, J. P. Sullivan, T. A. Friedmann, M. P. Siegal, T. W. Mercer and N. J. DiNardo, "A comparative study of residual stresses and microstructure in a-tC films", Mat. Res. Soc. Symp. Proc. 383, pp. 459-464, 1995.

12. M. P. Siegal, D. R. Tallant, L. J. Martinez-Miranda, N. J. DiNardo, J. P. Sullivan, P. Newcomer Provencio, J. C. Barbour, T. A. Friedmann, and R. L. Simpson, "Correlation of growth energetics with structural characterization of amorphous-tetrahedral diamond-like carbon films", unpublished.

13. J. Robertson, "Mechanical properties and coordinations of amorphous carbons", Phys. Rev. Lett. 68, pp. 220223, 1992.

14. V. I Merkulov, J. S. Lannin, C. H. Munro, S. A. Asher, V. S. Veerasamy, and W. I. Milne, "uv studies of tetrahedral bonding in diamondlike amorphous carbon", Phys. Rev. Lett. 78, pp. 4869-4872, 1997.

15. R. Lossy, D. L. Pappas, R. A. Roy, J. J. Cuomo, and V. M. Sura, "Filtered arc deposition of amorphous diamond", Appl. Phys. Lett. 61, pp. 171-173, 1992.

16. N. Missert, T. A. Friedmann, J. P. Sullivan, and R. G. Copeland, "Characterization of electron emission from planar amorphous carbon films using in situ scanning electron microscopy", Appl. Phys. Lett. 70, pp. 19951997, 1997.

17. J. P. Sullivan, P. B. Mirkarimi, K. F. McCarty, T. A. Friedmann, M. P. Siegal, and M. L. Lovejoy, "Electrical permittivities for thin film cubic boron nitride and hydrogen-free diamond-like carbon", unpublished.

18. B. S. Satyanarayana, A. Hart, W. I. Milne, and J. Robertson, "Field emission from tetrahedral amorphous carbon", Appl. Phys. Lett. 71, pp. 1430-1432, 1997.

19. Y. Lifshitz, S. R. Kasi, and J. W. Rabelais, "Subplantation model for film growth from hyperthermal species: application to diamond", Phys. Rev. Lett. 62, pp. 1290-1293, 1989. 
20. P. A. Schultz and E. B. Stechel, "Effects of basis quality on the prediction of structures, energies, and properties of amorphous tetrahedral carbon", Phys. Rev. B, 57, pp. 3295-3304, 1998.

21. N. A. Marks, D. R. McKenzie, B. A. Pailthorpe, M. Bernasconi, and M. Parrinello, "Microscopic structure of tetrahedral amorphous carbon", Phys. Rev. Lett. 76, pp. 768-771, 1996.

22. C. Z. Wang and K. M. Ho, "Structure, dynamics, and electronic properties of diamondlike amorphous carbon", Phys. Rev. Lett. 71, pp. 1184-1187, 1993.

23. D. A. Drabold, P. A. Fedders, and P. Stumm, "Theory of diamond-like amorphous carbon", Phys. Rev. B, 49, pp. 16415-16422, 1994.

24. J. P. Sullivan, T. A. Friedmann, D. R. Tallant, J. Mikkalson, D. J. Rieger, A. G. Baca, and L. J. MartinezMiranda, "Stress relief in pulsed-laser deposited amorphous tetrahedrally-bonded carbon films", Appl. Phys. Lett. in press.

25. L. J. Martinez-Miranda, J. P. Sullivan, T. A. Friedmann, M. P. Siegal, and N. J. DiNardo, "Structural characterization of the thermal evolution of tetrahedrally coordinated amorphous carbon films", Mat. Res. Soc. Symp. Proc. 498, 1998.

26. J. E. Jaskie, "Diamond-based field-emission displays", MRS Bulletin, 21, pp. 59-64, 1996.

27. A. A. Talin, T. E. Felter, T. A. Friedmann, J. P. Sullivan, and M. P. Siegal, "Electron field-emission from amorphous tetrahedrally bonded carbon-films", J. Vac. Sci. Technol. A, 13, pp. 1719-1722, 1996.

28. T. W. Mercer, N. J. DiNardo, J. B. Rothman, M. P. Siegal, T. A. Friedmann, and L. J. Martinez-Miranda, "Electron emission induced modifications in amorphous tetrahedral diamondlike carbon", Appl. Phys. Lett. 72, 1998.

29. D. R. Tallant, J. E. Parmeter, M. P. Siegal and R. L. Simpson, "The thermal stability of diamond-like carbon", Diamond and Related Mat. 4, pp. 191-199, 1995.

30. TRIMRC computer code by D. K. Brice, Albuquerque, NM.

31. M. P. Siegal, J. C. Barbour, P. Newcomer Provencio, D. R. Tallant, and T. A. Friedmann, "Amorphoustetrahedral diamond-like carbon layered structures resulting from film growth energetics", Appl. Phys. Lett. (submitted).

32. J. J. Cuomo, D. L. Pappas, J. Bruley, J. P. Doyle, and K. L. Saenger, "Vapor deposition processes for amorphous carbon films with $\mathrm{sp}^{3}$ fractions approaching diamond", J. Appl. Phys. 70, pp. 1706-1711, 1991.

33. P. H. Gaskell, A. Saeed, P. Chieux, and D. R. McKenzie, "Neutron-scattering studies of the structure of highly tetrahedral amorphous diamondlike carbon", Phys. Rev. Lett. 67, pp. 1286-1289, 1991.

34. D. L. Pappas, D. L. Saenger, J. Bruley, W. Krakow, J. J. Cuomo, T. Gu, and R. W. Collins, "Pulsed laser deposition of diamond-like carbon films", J. Appl. Phys. 71, pp. 5675-5684, 1992.

35. F. Xiong, Y. Y. Wang, V. Leppert, and R. P. H. Chang, "Pulsed laser deposition of amorphous diamond-like carbon films with ArF (193 nm) excimer laser", J. Mater. Res. 8, pp. 2265-2272, 1993.

36. M. F. Toney and S. Brennan, "Measurements of carbon thin-films using x-ray reflectivity", J. Appl. Phys. 66, pp. 1861-1863, 1989.

37. C. A. Lucas, T. D. Nguyen, and J. B. Kortright, "X-ray reflectivity measurements of the expansion of carbonfilms upon annealing”, Appl. Phys. Lett. 59, pp. 2100-2102, 1991.

38. D. R. Tallant, T. A. Friedmann, N. Missert, M. Siegal, and J. Sullivan, "Raman spectroscopy of amorphous carbon", Mat. Res. Soc. Symp. Proc. 498, 1998.

39. W. R. Gilkes, H. S. Sands, D. N. Batchelder, J. Robertson, and W. I. Milne, "Direct observation of $\mathrm{sp}^{3}$ bonding in tetrahedral amorphous-carbon using ultraviolet Raman spectroscopy", Appl. Phys. Lett. 70, pp. 1980-1982, 1997.

40. T. A. Friedmann, D. R. Tallant, J. P. Sullivan, M. P. Siegal, and R. L. Simpson, "Ion-assisted pulsed-laser deposition of amorphous tetrahedral-coordinated carbon films", Mat. Res. Soc. Symp. Proc. 1995.

41. T. E. Doyle and J. R. Dennison, "Vibrational dynamics and structure of graphitic amorphous-carbon modeled using an embedded-ring approach", Phys. Rev. B, 51, pp. 196-200, 1995.

42. A. G. Rinzler, J. H. Hafner, P. Nikolaev, L. Lou, S. G.Kim, D. Tomanid, P. Nordlander, D. T. Colbert, and R. E. Smalley, "Unraveling nanotubes: field emission from an atomic wire", Science, 269, pp. 1550-1553, 1995.

43. H. Schmid and H.-W. Fink, "Carbon nanotubes are coherent electron sources", Appl. Phys. Lett. 70, pp. 2679$2680,1997$.

44. Q. H. Wang, T. D. Corrigan, J. Y. Dai, R. P. H. Chang, and A. R. Krauss, "Field emission from nanotube bundle emitters at low fields", Appl. Phys. Lett. 70, pp. 3308-3310, 1997.

45. B. F. Coll, J. E. Jaskie, J. L. Markham, E. P. Menu, A. A. Talin, and P. von Allmen, "Field emission properties of disordered and partially ordered nanoclustered carbon films", Mat. Res. Soc. Symp. Proc. 498, 1998. 
M98005917

||||||||||||||||||||||||||||||||||||||||
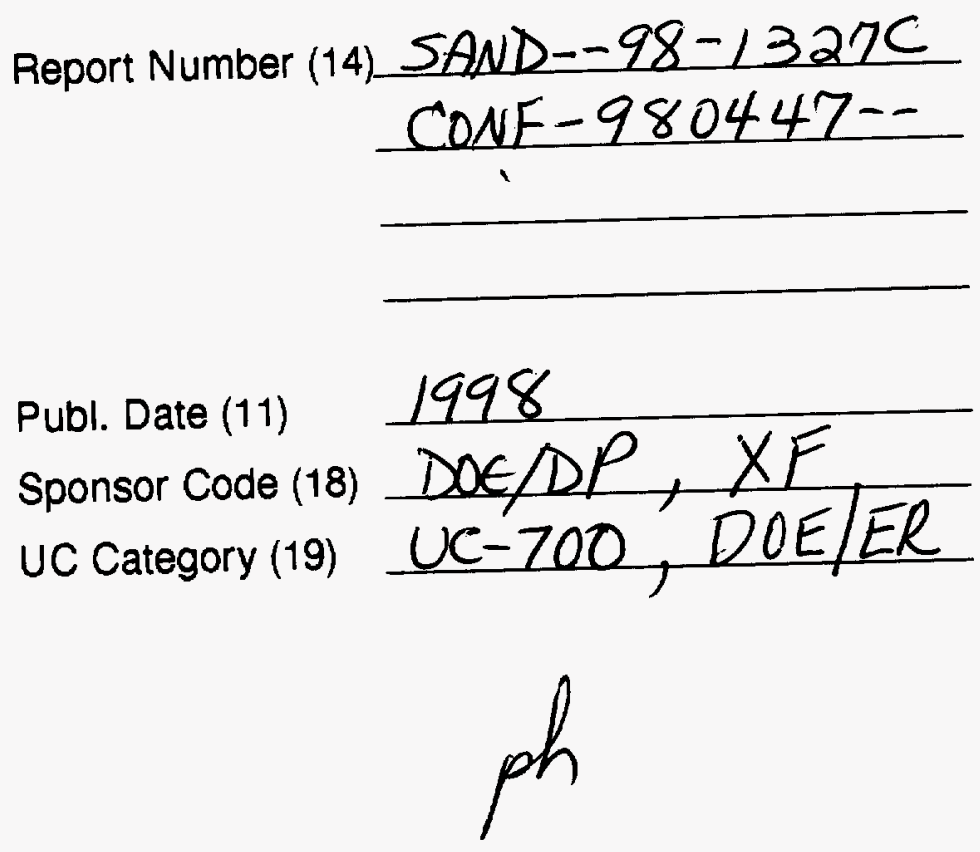

19980720070

DOE 